\title{
Photo-induced reductive cross-coupling of aldehydes, ketones and imines with electron-deficient arenes to construct aryl substituted alcohols and amines
}

\author{
Zan Liu a,b, Xiaolei Nan a,b, Tao Lei a,b, Chao Zhou ${ }^{\text {a,b, }}$, Yang Wang a,b, Wenqiang Liu a,b, Bin Chen a,b, \\ Chenho Tung a,b, Lizhu Wu ${ }^{a, b, *}$ \\ a Key Laboratory of Photochemical Conversion and Optoelectronic Materials, Technical Institute of Physics and Chemistry, Chinese Academy of Sciences, \\ Beijing 100190, China \\ b University of Chinese Academy of Sciences, Beijing 100049, China
}

\section{A R T I C L E I N F O}

\section{Article history:}

Received 17 September 2017

Accepted 26 October 2017

Published 5 March 2018

\section{Keywords:}

Aryl substituted alcohols and amines

Radical-radical cross coupling

Polarity reversal

Photocatalysis

Arylation

\begin{abstract}
A B S T R A C T
Umpolung reactions of $\mathrm{C}=\mathrm{X}$ bonds $(\mathrm{X}=\mathrm{O}, \mathrm{N})$ are valuable ways of constructing new $\mathrm{C}-\mathrm{C}$ bonds, which are sometimes difficult to be constructed using traditional synthetic pathways. Classical polarity inversion of $\mathrm{C}=\mathrm{X}$ bonds $(\mathrm{X}=\mathrm{O}, \mathrm{N})$ usually requires air or moisture-sensitive and strong reducing agents, which limit the feasibility of substrate scope. Herein we describe a photo-induced reductive cross-coupling reaction of aldehydes, ketones and imines with electron-deficient arenes (aromatic nitriles) using $f a c$-Ir(ppy) $)_{3}$ as a photocatalyst and diisopropylethylamine (DIPEA) as a terminal reductant under visible light irradiation. Mild conditions and high yields mean that this new polarity inversion strategy can be used with aryl-substituted alcohols and amines. Spectroscopic studies and control experiments have demonstrated the oxidative quenching of $\operatorname{Ir}(\mathrm{ppy})_{3}{ }^{*}$ by electron-deficient arenes involved in the key step for the $\mathrm{C}-\mathrm{C}$ bond formation.
\end{abstract}

(C) 2018, Dalian Institute of Chemical Physics, Chinese Academy of Sciences. Published by Elsevier B.V. All rights reserved.

\section{Introduction}

Substantial development in visible-light catalysis has been witnessed over recent years [1-3]. Due to the unique ability of visible-light harvesting, Ru(II) [4-11] and Ir(III) [12-17] complexes, organic dyes [18-21] and semiconductors [22,23] have been successfully used in single electron transfer (SET) to activate a variety of functional groups. In this regard, the umpolung conversion of $\mathrm{C}=\mathrm{X}$ double bonds $(\mathrm{X}=\mathrm{O}, \mathrm{N})$ is highly attractive. Classical polarity inversion of aldehydes, ketones and imines usually requires excess metals or unstable strong reducing agents under harsh conditions, meaning that only a narrow range of substrates are compatible [24,25], greatly limiting their subsequent transformation. In contrast to using traditional methods, these $\mathrm{C}=\mathrm{X}$ double bonds $(\mathrm{X}=\mathrm{O}, \mathrm{N})$ can easily obtain one electron via visible-light catalysis to be converted into a practical nucleophilic intermediate. In 2013, Knowles et al. $[26,27]$ reported an intramolecular reductive coupling between ketones and hydrazones or electron-deficient olefins under visible-light catalysis via proton coupled electron transfer. In the same year, MacMillan et al. [28,29] demonstrated the coupling of ketones and amines with activated $\mathrm{C}-\mathrm{H}$ bonds, including benzylic ethers with the $\beta$-position of enamines generated in situ. More recently, Rueping et al. [30,31] realized the dimer-

\footnotetext{
*Corresponding author. Tel/Fax: +86-10-82543580; E-mail: lzwu@mail.ipc.ac.cn

This work was supported by the Ministry of Science and Technology of China (2013CB834804, 2014CB239402), the National Natural Science Foundation of China (21390404, 91427303), and the Strategic Priority Research Program of the Chinese Academy of Science (XDB17030400). DOI: 10.1016/S1872-2067(17)62896-1 | http://www.sciencedirect.com/science/journal/18722067 | Chin. J. Catal., Vol. 39, No. 3, March 2018
} 
ization of $\mathrm{C}=\mathrm{X}$ double bonds $(\mathrm{X}=\mathrm{O}, \mathrm{N})$ and the cross coupling of tertiary amines and ketones assisted by photo-induced two-center/three-electron intermediates. Xiao et al. [32] expanded this cross coupling reaction to include secondary amines. Chen et al. [33] demonstrated the visible-light-induced polarity-reversed allylation of aldehydes, ketones and imines with electron-withdrawing allyl sulfones. Furthermore, Ngai et al. [34] described reductive coupling of these polar $\mathrm{C}=\mathrm{X}$ double bonds $(\mathrm{X}=\mathrm{O}, \mathrm{N})$ with weak electrophilic alkenylpyridines through chelation with a lanthanide. Visible-light-induced polarity inversion of $\mathrm{C}=\mathrm{X}$ bonds $(\mathrm{X}=\mathrm{O}, \mathrm{N})$ has demonstrated the potential to synthesize alkyl alcohols and amines. We were keen to investigate if a visible-light strategy could be applied to the construction of aryl-substituted products, which are generally synthesized using air- or water-sensitive Grignard reagents under thermal conditions (Scheme 1). This would provide a simple and mild way to generate aryl alcohols and amines, with no need for harsh conditions and sensitive reagents.

\section{Experimental}

\subsection{General methods}

${ }^{1} \mathrm{H}$ NMR spectra were recorded using a Bruker Avance DPX $400 \mathrm{MHz}$ instrument with tetramethylsilane (TMS) as the internal standard. ${ }^{13} \mathrm{C}$ NMR spectra were obtained at $100 \mathrm{MHz}$ and referenced to the internal solvent signals. Mass spectra were recorded using a Trio-2000 GC-MS spectrometer. Commercially available reagents and solvents were used without further purification. Blue LEDs $(3 \mathrm{~W}, \lambda=450 \pm 10 \mathrm{~nm}, 145 \mathrm{~lm} @$ $700 \mathrm{~mA}$ ) were used as the irradiation light.

\subsection{General procedure for the preparation of imines}

The aldehyde (1.0 mmol) was added to a solution of aryl amine $(1.0 \mathrm{mmol})$ in anhydrous $\mathrm{Et}_{2} \mathrm{O}(20 \mathrm{~mL})$ in the presence of molecular sieves ( $4 \AA 1.6 \mathrm{~mm}$ pellets, $7 \mathrm{~g}$ ). The reaction was performed at room temperature (RT) under magnetic stirring and monitored by TLC. When the reaction was complete, the molecular sieves were removed by filtration, and the solvent was removed under vacuum. The residue was recrystallized from $\mathrm{Et}_{2} \mathrm{O} / n$-hexane to afford the imines directly as pure $(E)$ products.

\subsection{General procedure for radical trapping experiments}

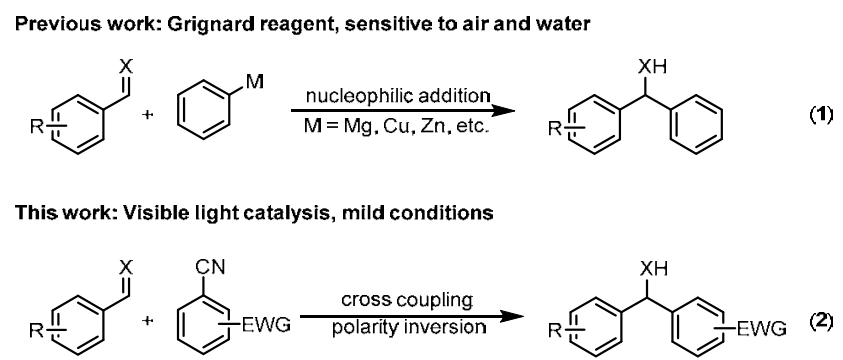

Scheme 1. Visible light catalyzed umpolung reactions of $C=X(X=0, N)$ with electron-deficient arenes.
A 10-mL Pyrex tube equipped with a magnetic stir bar was charged with 1,4-dicyanobenzene (1,4-DCB) (25.6 mg, 0.2 mmol), fac-Ir(ppy)3 (2.6 mg, $2 \mathrm{~mol} \%$ ), TEMPO (0.4 mmol, 2 equiv.) and DMSO (2 $\mathrm{mL})$. The Pyrex tube was sealed with rubber plug and then deaerated by bubbling Ar for $15 \mathrm{~min}$. benzaldehyde ( $30.5 \mu \mathrm{L}, 0.3 \mathrm{mmol})$ and DIPEA $(52.4 \mu \mathrm{L}, 0.3$ mmol) were added. The reaction system was irradiated with blue LEDs $(\lambda=450 \pm 10 \mathrm{~nm}$ ) for $12 \mathrm{~h}$ at RT. When the reaction was complete, the aqueous solution was extracted with ethyl acetate $(5 \mathrm{~mL} \times 3)$. The organic extracts were combined, washed with brine and dried over anhydrous sodium sulfate. The solvent was removed under vacuum, then diphenylacetonitrile $(23.2 \mathrm{mg}$ ) was added as an internal standard and the yield (19\%) was detected by ${ }^{1} \mathrm{H}$ NMR.

\subsection{General procedure for the polarity inversion/arylation process}

A 10-mL Pyrex tube equipped with a magnetic stir bar was charged with 1,4-DCB (25.6 mg, $0.2 \mathrm{mmol})$, fac-Ir(ppy) 3 (2.6 $\mathrm{mg}, 2 \mathrm{~mol} \%$ ) and DMSO (2 mL). The Pyrex tube was sealed with rubber plug and then deaerated by bubbling Ar for $15 \mathrm{~min}$, then benzaldehyde ( $30.5 \mu \mathrm{L}, 0.3 \mathrm{mmol}$ ) and DIPEA (52.4 $\mu \mathrm{L}, 0.3$ mmol) were added. The reaction system was irradiated with blue LEDs $(\lambda=450 \pm 10 \mathrm{~nm})$ for $12 \mathrm{~h}$ at RT. When the reaction was complete, the aqueous solution was extracted with ethyl acetate $(5 \mathrm{~mL} \times 3)$. The organic extracts were combined, washed with brine and dried over anhydrous sodium sulfate. The solvent was removed under vacuum and the residue was purified by chromatography on silica gel (petroleum ether: ethyl acetate $=3: 1$ ) to afford the desired product.

4-(Hydroxy(phenyl)methyl)benzonitrile (3): colorless oil, isolated yield: $83 \% .{ }^{1} \mathrm{H}$ NMR $\left(400 \mathrm{MHz}, \mathrm{CDCl}_{3}\right) \delta 7.59(\mathrm{~d}, J=8.4$ $\mathrm{Hz}, 2 \mathrm{H}$ ), 7.49 (d, J = 8.2 Hz, 2H), 7.38-7.26 (m, 5H), 5.83 (s, 1H), 2.65 (s, 1H). ${ }^{13} \mathrm{C}$ NMR (100 MHz, $\left.\mathrm{CDCl}_{3}\right)$ 149.01, 142.93, 132.34, 128.96, 128.36, 127.12, 126.78, 118.91, 111.18, 75.69. HRMS (EI) calculated for $\mathrm{C}_{14} \mathrm{H}_{11} \mathrm{NO}[\mathrm{M}-\mathrm{H}]^{+}$: 208.0762, found: 208.0761.

4-(Hydroxy(phenyl)methyl)-2-methylbenzonitrile and 4-(hydroxyl(phenyl)methyl)-3-methylbenzonitrile (4): colorless oil, isolated yield: $82 \%$, the ratio of regioselectivity $=1.47$. ${ }^{1} \mathrm{H}$ NMR (400 MHz, $\left.\mathrm{CDCl}_{3}\right) \delta 7.76(\mathrm{~d}, J=8.0 \mathrm{~Hz}, 0.40 \mathrm{H}$ ), 7.58-7.50 (m, $1.00 \mathrm{H}), 7.41-7.21(\mathrm{~m}, 6.60 \mathrm{H}), 5.95 / 5.80(\mathrm{~s}$, $1.00 \mathrm{H}), 2.53(\mathrm{~d}, J=2.7 \mathrm{~Hz}, 0.60 \mathrm{H}), 2.50(\mathrm{~s}, 1.80 \mathrm{H}), 2.45(\mathrm{~d}, J=$ $3.1 \mathrm{~Hz}, 0.40 \mathrm{H}), 2.20$ (s, $1.20 \mathrm{H}) .{ }^{13} \mathrm{C}$ NMR $\left(100 \mathrm{MHz}, \mathrm{CDCl}_{3}\right) \delta$ $148.80,146.78,143.04,142.28,141.65,136.64,133.87,132.74$, $130.04,128.94,128.93,128.40,128.30,128.11,127.40,126.92$, $126.75,124.36,119.06,118.22,111.68,111.22,75.75,73.22$, 20.66, 19.33. HRMS (EI) calculated for $\mathrm{C}_{15} \mathrm{H}_{13} \mathrm{NO}[\mathrm{M}]^{+}$: 223.0997, found: 223.0999.

Phenyl(4-(phenylsulfonyl)phenyl)methanol (5): white solid, isolated yield: $52 \% .{ }^{1} \mathrm{H}$ NMR $\left(400 \mathrm{MHz}, \mathrm{CDCl}_{3}\right) \delta 7.97-7.79(\mathrm{~m}$, $4 \mathrm{H}), 7.54-7.45(\mathrm{~m}, 5 \mathrm{H}), 7.37-7.22(\mathrm{~m}, 5 \mathrm{H}), 5.83(\mathrm{~s}, 1 \mathrm{H}), 2.59(\mathrm{~s}$, 1H). ${ }^{13} \mathrm{C}$ NMR $\left(100 \mathrm{MHz}, \mathrm{CDCl}_{3}\right) \delta 149.46,142.96,141.68$, 140.52, 133.30, 129.39, 128.92, 128.29, 127.94, 127.76, 127.33, 126.77, 75.69. HRMS (ESI) calculated for $\mathrm{C}_{19} \mathrm{H}_{16} \mathrm{O}_{3} \mathrm{~S}[\mathrm{M}+\mathrm{Na}]^{+}$: 347.0712, found: 347.0709 . 
Methyl 4-(hydroxy(phenyl)methyl)benzoate (6): colorless oil, isolated yield: $48 \% .{ }^{1} \mathrm{H}$ NMR $\left(400 \mathrm{MHz}, \mathrm{CDCl}_{3}\right) \delta 7.97(\mathrm{~d}, J=$ $8.3 \mathrm{~Hz}, 2 \mathrm{H}), 7.44(\mathrm{~d}, J=8.2 \mathrm{~Hz}, 2 \mathrm{H}), 7.34-7.24(\mathrm{~m}, 5 \mathrm{H}), 5.83(\mathrm{~s}$, 1H), 3.87 (s, 3H), 2.68 (s, 1H). ${ }^{13} \mathrm{C}$ NMR (100 MHz, $\left.\mathrm{CDCl}_{3}\right) \delta$ 167.07, 148.89, 143.41, 129.88, 129.32, 128.78, 128.03, 126.77, 126.45, 75.99, 52.19. HRMS (EI) calculated for $\mathrm{C}_{15} \mathrm{H}_{14} \mathrm{O}_{3}[\mathrm{M}]^{+}$: 242.0943, found: 242.0941 .

5-(Hydroxy(phenyl)methyl)isobenzofuran-1(3H)-one (7): white solid, isolated yield: $42 \% .{ }^{1} \mathrm{H}$ NMR $\left(400 \mathrm{MHz}^{\mathrm{CDCl}} 3\right) \delta$ $7.80(\mathrm{~d}, J=7.9 \mathrm{~Hz}, 1 \mathrm{H}), 7.55(\mathrm{~s}, 1 \mathrm{H}), 7.51(\mathrm{~d}, J=7.9 \mathrm{~Hz}, 1 \mathrm{H})$, 7.35-7.35 (m, 5H), 5.92 (s, 1H), $5.24(\mathrm{~s}, 2 \mathrm{H}), 2.94(\mathrm{~s}, 1 \mathrm{H}) .{ }^{13} \mathrm{C}$ $\mathrm{NMR}\left(100 \mathrm{MHz}, \mathrm{CDCl}_{3}\right) \delta 171.14,150.88,147.13,143.16$, 128.93, 128.29, 127.70, 126.76, 125.75, 124.81, 119.83, 75.90, 69.79. HRMS (EI) calculated for $\mathrm{C}_{15} \mathrm{H}_{12} \mathrm{O}_{3}$ [M]+: 240.0786, found: 240.0790 .

Phenyl(1H-pyrrolo[2,3-b]pyridin-4-yl)methanol (8): yellow oil, isolated yield: $82 \% .{ }^{1} \mathrm{H}$ NMR (400 MHz, $\left.\mathrm{CDCl}_{3}\right) \delta 10.50(\mathrm{~s}$, $1 \mathrm{H}), 8.13(\mathrm{~d}, J=5.0 \mathrm{~Hz}, 1 \mathrm{H}), 7.37(\mathrm{~d}, J=7.1 \mathrm{~Hz}, 2 \mathrm{H}), 7.26-7.09$ (m, 5H), $6.38(\mathrm{~d}, J=3.5 \mathrm{~Hz}, 1 \mathrm{H}), 6.12(\mathrm{~s}, 1 \mathrm{H}), 3.37(\mathrm{~s}, 1 \mathrm{H}) .{ }^{13} \mathrm{C}$ NMR $\left(100 \mathrm{MHz}, \mathrm{CDCl}_{3}\right) \delta 148.83,145.19,142.85,128.74$, 128.07, 126.98, 125.13, 118.06, 112.84, 99.84, 74.49. HRMS (ESI) calculated for $\mathrm{C}_{14} \mathrm{H}_{12} \mathrm{~N}_{2} \mathrm{O}[\mathrm{M}+\mathrm{H}]^{+}$: 225.1028, found: 225.1016.

Phenyl(pyridin-4-yl)methanol (9): yellow solid, isolated yield: $99 \% .{ }^{1} \mathrm{H}$ NMR $\left(400 \mathrm{MHz}, \mathrm{CDCl}_{3}\right) \delta 8.25(\mathrm{dd}, J=4.6,1.6 \mathrm{~Hz}$, 2H), 7.30-7.23 (m, 7H), 5.70 (s, 1H). ${ }^{13} \mathrm{C}$ NMR (100 MHz, $\left.\mathrm{CDCl}_{3}\right)$ $\delta 153.83,149.05,143.16,128.71,128.01,126.87,121.53,74.59$. HRMS (ESI) calculated for $\mathrm{C}_{12} \mathrm{H}_{11} \mathrm{NO}[\mathrm{M}+\mathrm{H}]+:$ 186.0919, found: 186.0911.

(2,6-Dimethylpyridin-4-yl)(phenyl)methanol (10): yellow oil, isolated yield: $99 \% .{ }^{1} \mathrm{H}$ NMR $\left(400 \mathrm{MHz}, \mathrm{CDCl}_{3}\right) \delta 7.48-7.20$ (m, 5H), $6.96(\mathrm{~s}, 2 \mathrm{H}), 5.68(\mathrm{~s}, 1 \mathrm{H}), 4.05(\mathrm{~s}, 1 \mathrm{H}), 2.42(\mathrm{~s}, 6 \mathrm{H}) .{ }^{13} \mathrm{C}$ NMR $\left(100 \mathrm{MHz}, \mathrm{CDCl}_{3}\right) \delta 157.81,153.63,143.25,128.75$, $128.06,126.85,118.07,74.94,24.30$. HRMS (ESI) calculated for $\mathrm{C}_{14} \mathrm{H}_{15} \mathrm{NO}[\mathrm{M}+\mathrm{H}]+:$ : 214.1232, found: 214.1224.

Isoquinolin-1-yl(phenyl)methanol (11): yellow oil, isolated yield: $86 \% .{ }^{1} \mathrm{H}$ NMR (400 MHz, $\left.\mathrm{CDCl}_{3}\right) \delta 8.53(\mathrm{~d}, J=5.6 \mathrm{~Hz}, 1 \mathrm{H})$, $7.95(\mathrm{~d}, J=8.4 \mathrm{~Hz}, 1 \mathrm{H}), 7.82(\mathrm{~d}, J=8.1 \mathrm{~Hz}, 1 \mathrm{H}), 7.64-7.60(\mathrm{~m}$, 2H), 7.49-7.45 (m, 1H), 7.41-7.16 (m, 5H), $6.36(\mathrm{~s}, 1 \mathrm{H}), 6.15(\mathrm{~s}$, 1H). ${ }^{13} \mathrm{C} \mathrm{NMR}\left(100 \mathrm{MHz}, \mathrm{CDCl}_{3}\right) \delta 159.33,143.47,140.14$, $136.71,130.38,128.83,127.97,127.79,127.57,127.50,125.34$, 124.92, 121.20, 72.70. HRMS (ESI) calculated for $\mathrm{C}_{16} \mathrm{H}_{13} \mathrm{NO}$ $[\mathrm{M}+\mathrm{H}]^{+}:$236.1075, found: 236.1067 .

4-((4-Chlorophenyl)(hydroxy)methyl)benzonitrile (12): white solid, isolated yield: $55 \% .{ }^{1} \mathrm{H}$ NMR (400 $\left.\mathrm{MHz}, \mathrm{CDCl}_{3}\right) \delta$ $7.61(\mathrm{~d}, J=8.1 \mathrm{~Hz}, 2 \mathrm{H}), 7.48$ (d, $J=8.1 \mathrm{~Hz}, 2 \mathrm{H}), 7.32$ (d, $J=8.4$ $\mathrm{Hz}, 2 \mathrm{H}$ ), 7.27 (d, $J=7.7 \mathrm{~Hz}, 2 \mathrm{H}), 5.84$ (s, 1H), 2.55 (s, 1H). ${ }^{13} \mathrm{C}$ NMR $\left(100 \mathrm{MHz}, \mathrm{CDCl}_{3}\right) \delta 148.55,141.35,134.22,132.50$, $129.13,128.14,127.13,118.79,111.56,75.05$. HRMS (ESI) calculated for $\mathrm{C}_{14} \mathrm{H}_{10} \mathrm{ClNO}[\mathrm{M}-\mathrm{H}]+:$ : 242.0373, found: 242.0377 .

4-(Hydroxy(4-methoxyphenyl)methyl)benzonitrile (13): white solid, isolated yield: $83 \%$. ${ }^{1} \mathrm{H}$ NMR $\left(400 \mathrm{MHz}, \mathrm{CDCl}_{3}\right) \delta$ $7.60(\mathrm{~d}, J=8.1 \mathrm{~Hz}, 2 \mathrm{H}), 7.49$ (d, $J=8.0 \mathrm{~Hz}, 2 \mathrm{H}), 7.22$ (d, $J=8.4$ $\mathrm{Hz}, 2 \mathrm{H}), 6.86$ (d, J = 8.4 Hz, 2H), 5.81 (s, 1H), 3.78 (s, 3H), 2.45 (s, $1 \mathrm{H}) .{ }^{13} \mathrm{C}$ NMR $\left(100 \mathrm{MHz}, \mathrm{CDCl}_{3}\right) \delta 159.66,149.26,135.24$, 132.31, 128.21, 127.03, 118.97, 114.35, 111.10, 75.30, 55.43. HRMS (ESI) calculated for $\mathrm{C}_{15} \mathrm{H}_{13} \mathrm{NO}_{2}[\mathrm{M}-\mathrm{H}]^{+}:$238.0863, found:
238.0871.

4-(Hydroxy(thiophen-2-yl)methyl)benzonitrile (14): yellow oil, isolated yield: $68 \% .{ }^{1} \mathrm{H}$ NMR $\left(400 \mathrm{MHz}, \mathrm{CDCl}_{3}\right) \delta 7.64(\mathrm{~d}, J=$ $8.1 \mathrm{~Hz}, 2 \mathrm{H}), 7.56(\mathrm{~d}, J=8.2 \mathrm{~Hz}, 2 \mathrm{H}), 7.29$ (d, $J=5.0 \mathrm{~Hz}, 1 \mathrm{H})$, 6.98-6.94 (m, 1H), 6.93-6.92 (m, 1H), $6.10(\mathrm{~s}, 1 \mathrm{H}), 2.76(\mathrm{~d}, J=$ $17.4 \mathrm{~Hz}, 1 \mathrm{H}) .{ }^{13} \mathrm{C}$ NMR $\left(100 \mathrm{MHz}, \mathrm{CDCl}_{3}\right) \delta 148.23,146.83$, $132.44,126.99,126.31,125.56,118.83,111.67,71.53$. HRMS (EI) calculated for $\mathrm{C}_{12} \mathrm{H}_{9} \mathrm{NOS}[\mathrm{M}]^{+}: 215.0405$, found: 215.0408 .

4-(Hydroxy(naphthalen-2-yl)methyl)benzonitrile (15): white solid, isolated yield: $62 \%$. ${ }^{1} \mathrm{H}$ NMR $\left(400 \mathrm{MHz}, \mathrm{CDCl}_{3}\right) \delta$ 7.87-7.79 (m, 4H), 7.66-7.44 (m, 6H), $7.37(\mathrm{~m}, 1 \mathrm{H}), 6.02(\mathrm{~s}$, $1 \mathrm{H}), 2.52(\mathrm{~s}, 1 \mathrm{H}) .{ }^{13} \mathrm{C} \mathrm{NMR}\left(100 \mathrm{MHz}, \mathrm{CDCl}_{3}\right) \delta 148.78,140.19$, 133.32, 133.24, 132.41, 129.02, 128.16, 127.88, 127.26, 126.68, $126.57,125.74,124.48,118.92,111.35,75.88$. HRMS (EI) calculated for $\mathrm{C}_{18} \mathrm{H}_{13} \mathrm{NO}$ [M]+: 259.0997, found: 259.0994 .

4-(1-Hydroxy-1-phenylethyl)benzonitrile (18): colorless oil, isolated yield: $82 \% .{ }^{1} \mathrm{H}$ NMR $\left(400 \mathrm{MHz}, \mathrm{CDCl}_{3}\right) \delta 7.58(\mathrm{~d}, J=8.2$ $\mathrm{Hz}, 2 \mathrm{H}), 7.53$ (d, J= 8.2 Hz, 2H), 7.42-7.23 (m, 5H), 2.38 (s, 1H), 1.95 (s, 3H). ${ }^{13} \mathrm{C}$ NMR $\left(100 \mathrm{MHz}, \mathrm{CDCl}_{3}\right) \delta 153.45,146.75$, 132.13, 128.65, 127.74, 126.66, 125.93, 118.96, 110.77, 76.08, 30.66. HRMS (EI) calculated for $\mathrm{C}_{15} \mathrm{H}_{13} \mathrm{NO}$ [M]+: 223.0997, found: 223.0995.

4-(1-(4-Ethylphenyl)-1-hydroxyethyl)benzonitrile colorless oil, isolated yield: $87 \% .{ }^{1} \mathrm{H}$ NMR $\left(400 \mathrm{MHz}, \mathrm{CDCl}_{3}\right) \delta$ 7.58 (d, $J=8.4 \mathrm{~Hz}, 2 \mathrm{H}$ ), 7.53 (d, $J=8.4 \mathrm{~Hz}, 2 \mathrm{H}$ ), 7.29 (d, $J=8.1$ $\mathrm{Hz}, 2 \mathrm{H}), 7.17$ (d, $J=8.1 \mathrm{~Hz}, 2 \mathrm{H}), 2.63(\mathrm{q}, J=7.6 \mathrm{~Hz}, 2 \mathrm{H}), 2.26(\mathrm{~s}$, 1H), 1.93 (s, 3H), 1.22 (t, $J=7.6 \mathrm{~Hz}, 3 \mathrm{H}) .{ }^{13} \mathrm{C}$ NMR $(100 \mathrm{MHz}$, $\left.\mathrm{CDCl}_{3}\right) \delta 153.68,144.08,143.87,132.10,128.13,126.64$, $125.95,119.03,110.68,76.00,30.72,28.51,15.53$. HRMS (MALDI-TOF) calculated for $\mathrm{C}_{17} \mathrm{H}_{17} \mathrm{NO}[\mathrm{M}+\mathrm{Na}]^{+:}$274.1208, found: 274.1202 .

4-(1-Hydroxy-2,3-dihydro-1H-inden-1-yl)benzonitrile (20): colorless oil, isolated yield: $81 \% .{ }^{1} \mathrm{H}$ NMR $\left(400 \mathrm{MHz} \mathrm{CDCl}_{3}\right) \delta$ $7.61(\mathrm{~d}, J=8.3 \mathrm{~Hz}, 2 \mathrm{H}), 7.51(\mathrm{~d}, J=8.3 \mathrm{~Hz}, 2 \mathrm{H}), 7.38-7.29(\mathrm{~m}$, 2H), 7.25-7.21 (m, 1H), $7.01(\mathrm{~d}, J=7.6 \mathrm{~Hz}, 1 \mathrm{H}), 3.23-3.21(\mathrm{~m}$, $1 \mathrm{H}), 3.06-2.93(\mathrm{~m}, 1 \mathrm{H}), 2.53-2.40(\mathrm{~m}, 2 \mathrm{H}), 2.21(\mathrm{~s}, 1 \mathrm{H}) .{ }^{13} \mathrm{C}$ NMR $\left(100 \mathrm{MHz}, \mathrm{CDCl}_{3}\right) \delta 152.03,147.19,144.21,132.05$, 129.21, 127.55, 126.70, 125.33, 123.99, 119.08, 110.82, 85.41, 44.98, 30.07. HRMS (EI) calculated for $\mathrm{C}_{16} \mathrm{H}_{13} \mathrm{NO}[\mathrm{M}]^{+}$: 235.0997, found: 235.0999 .

4-(1-(4-Chlorophenyl)-1-hydroxyethyl)benzonitrile (21): white solid, isolated yield: $76 \%$. ${ }^{1} \mathrm{H}$ NMR $\left(400 \mathrm{MHz}, \mathrm{CDCl}_{3}\right) \delta$ $7.60(\mathrm{~d}, J=8.3 \mathrm{~Hz}, 2 \mathrm{H}), 7.51(\mathrm{~d}, J=8.2 \mathrm{~Hz}, 2 \mathrm{H}), 7.31(\mathrm{~m}, 4 \mathrm{H})$, 2.35 (s, $1 \mathrm{H}), 1.94(\mathrm{~s}, 3 \mathrm{H}) .{ }^{13} \mathrm{C}$ NMR $\left(100 \mathrm{MHz}, \mathrm{CDCl}_{3}\right) \delta 152.86$, 145.31, 133.65, 132.26, 128.75, 127.43, 126.61, 118.83, 111.08, 75.72, 30.69. HRMS (EI) calculated for $\mathrm{C}_{15} \mathrm{H}_{12} \mathrm{ClNO}[\mathrm{M}]^{+}$: 257.0607 found: 257.0610 .

4-(1-Hydroxy-1-(4-methoxyphenyl)ethyl)benzonitrile (22): colorless oil, isolated yield: $76 \% .{ }^{1} \mathrm{H}$ NMR $\left(400 \mathrm{MHz} \mathrm{CDCl}_{3}\right) \delta$ $7.58(\mathrm{~d}, J=8.4 \mathrm{~Hz}, 2 \mathrm{H}), 7.52$ (d, $J=8.5 \mathrm{~Hz}, 2 \mathrm{H}), 7.29$ (d, $J=8.8$ $\mathrm{Hz}, 2 \mathrm{H}), 6.85$ (d, $J=8.8 \mathrm{~Hz}, 2 \mathrm{H}), 3.79(\mathrm{~s}, 3 \mathrm{H}), 2.29$ (d, $J=11.9 \mathrm{~Hz}$, 1H), 1.92 (s, 3H). ${ }^{13} \mathrm{C}$ NMR (100 MHz, $\left.\mathrm{CDCl}_{3}\right) \delta 159.07,153.80$, 139.00, 132.09, 127.30, 126.60, 119.02, 113.92, 110.64, 75.80, 55.41, 30.82. HRMS (EI) calculated for $\mathrm{C}_{16} \mathrm{H}_{15} \mathrm{NO}_{2}[\mathrm{M}]^{+}$: 253.1103, found: 253.1106.

4-(Cyclopropyl(hydroxy)(phenyl)methyl)benzonitrile (23): white solid, isolated yield: $67 \% .{ }^{1} \mathrm{H}$ NMR $\left(400 \mathrm{MHz}, \mathrm{CDCl}_{3}\right) \delta$ 
7.59 (d, $J=8.3 \mathrm{~Hz}, 2 \mathrm{H}), 7.53$ (d, $J=8.2 \mathrm{~Hz}, 2 \mathrm{H}), 7.44$ (d, $J=8.0$ $\mathrm{Hz}, 2 \mathrm{H}), 7.37-7.28$ (m, 3H), $1.96(\mathrm{~s}, 1 \mathrm{H}), 1.61-1.58(\mathrm{~m}, 1 \mathrm{H})$, 0.76-0.64 (m, 1H), 0.60-0.41 (m, 3H). ${ }^{13} \mathrm{C}$ NMR (100 MHz, $\left.\mathrm{CDCl}_{3}\right) \delta 152.78,146.16,131.89,128.49,127.90,127.49$, 127.07, 119.06, 110.82, 76.96, 21.54, 2.53, 1.38. HRMS (EI) calculated for $\mathrm{C}_{17} \mathrm{H}_{15} \mathrm{NO}[\mathrm{M}]^{+}: 249.1154$, found: 249.1156 .

4-(Phenyl(phenylamino)methyl)benzonitrile (24): pale yellow oil, isolated yield: $83 \% .{ }^{1} \mathrm{H}$ NMR $\left(400 \mathrm{MHz}, \mathrm{CDCl}_{3}\right) \delta 7.63(\mathrm{~d}$, $J=8.3 \mathrm{~Hz}, 2 \mathrm{H}), 7.55(\mathrm{~d}, J=8.1 \mathrm{~Hz}, 2 \mathrm{H}), 7.40-7.31(\mathrm{~m}, 5 \mathrm{H}), 7.16$ $(\mathrm{t}, J=7.6 \mathrm{~Hz}, 2 \mathrm{H}), 6.76(\mathrm{t}, J=7.3 \mathrm{~Hz}, 1 \mathrm{H}), 6.54(\mathrm{~d}, J=8.2 \mathrm{~Hz}, 2 \mathrm{H})$, $5.55(\mathrm{~s}, 1 \mathrm{H}), 4.26(\mathrm{~s}, 1 \mathrm{H}) .{ }^{13} \mathrm{C} \mathrm{NMR}\left(100 \mathrm{MHz}, \mathrm{CDCl}_{3}\right) \delta 148.30$, $146.83,141.93,132.69,129.35,129.21,128.18,128.08,127.71$, 118.87, 118.42, 113.65, 111.26, 63.01. HRMS (MALDI-TOF) calculated for $\mathrm{C}_{20} \mathrm{H}_{16} \mathrm{~N}_{2}[\mathrm{M}+\mathrm{H}]^{+}: 285.1392$, found: 285.1388 .

4-((Phenylamino)(p-tolyl)methyl)benzonitrile (25): pale yellow oil, isolated yield: $90 \% .{ }^{1} \mathrm{H}$ NMR $\left(400 \mathrm{MHz}, \mathrm{CDCl}_{3}\right) \delta 7.63$ (d, $J=8.3 \mathrm{~Hz}, 2 \mathrm{H}), 7.55(\mathrm{~d}, J=8.2 \mathrm{~Hz}, 2 \mathrm{H}), 7.24-7.12(\mathrm{~m}, 6 \mathrm{H})$, $6.77(\mathrm{t}, J=7.3 \mathrm{~Hz}, 1 \mathrm{H}), 6.54(\mathrm{~d}, J=7.9 \mathrm{~Hz}, 2 \mathrm{H}), 5.52(\mathrm{~s}, 1 \mathrm{H}), 4.26$ (s, $1 \mathrm{H}), 2.37$ (s, 3H). ${ }^{13} \mathrm{C}$ NMR (100 MHz, $\left.\mathrm{CDCl}_{3}\right) \delta 148.55$, 146.89, 139.08, 138.00, 132.66, 129.87, 129.34, 128.01, 127.62, 118.92, 118.36, 113.64, 111.16, 62.76, 21.18. HRMS (MALDI-TOF) calculated for $\mathrm{C}_{21} \mathrm{H}_{18} \mathrm{~N}_{2}[\mathrm{M}+\mathrm{H}]^{+}: 299.1548$, found: 299.1545.

4-((4-Methoxyphenyl)(phenylamino)methyl)benzonitrile (26): white solid, isolated yield: $98 \%$. ${ }^{1} \mathrm{H}$ NMR $(400 \mathrm{MHz}$, $\left.\mathrm{CDCl}_{3}\right) \delta 7.58(\mathrm{~d}, J=8.2 \mathrm{~Hz}, 2 \mathrm{H}), 7.49(\mathrm{~d}, J=8.1 \mathrm{~Hz}, 2 \mathrm{H}), 7.17(\mathrm{~d}$, $J=8.5 \mathrm{~Hz}, 2 \mathrm{H}), 7.11(\mathrm{t}, J=7.8 \mathrm{~Hz}, 2 \mathrm{H}), 6.85(\mathrm{~d}, J=8.5 \mathrm{~Hz}, 2 \mathrm{H})$, $6.71(\mathrm{t}, J=7.4 \mathrm{~Hz}, 1 \mathrm{H}), 6.49(\mathrm{~d}, J=7.9 \mathrm{~Hz}, 2 \mathrm{H}), 5.46(\mathrm{~s}, 1 \mathrm{H}), 4.19$ (s, $1 \mathrm{H}), 3.76$ (s, 3H). ${ }^{13} \mathrm{C}$ NMR (100 MHz, $\left.\mathrm{CDCl}_{3}\right) \delta 159.40$, 148.63, 146.87, 134.14, 132.64, 129.31, 128.89, 127.98, 118.91, 118.30, 114.51, 113.61, 111.09, 62.34, 55.38. HRMS (MALDI-TOF) calculated for $\mathrm{C}_{21} \mathrm{H}_{18} \mathrm{~N} 2 \mathrm{O}[\mathrm{M}-\mathrm{H}]^{+}$: 313.1341 , found: 313.1337.

4-((4-Chlorophenyl)(phenylamino)methyl)benzonitrile (27): white solid, isolated yield: $82 \%$. ${ }^{1} \mathrm{H}$ NMR $(400 \mathrm{MHz}$, $\left.\mathrm{CDCl}_{3}\right) \delta 7.61(\mathrm{~d}, J=8.3 \mathrm{~Hz}, 2 \mathrm{H}), 7.47(\mathrm{~d}, J=8.2 \mathrm{~Hz}, 2 \mathrm{H}), 7.31(\mathrm{~d}$, $J=8.5 \mathrm{~Hz}, 2 \mathrm{H}), 7.23(\mathrm{~d}, J=8.4 \mathrm{~Hz}, 2 \mathrm{H}), 7.17-7.09(\mathrm{~m}, 2 \mathrm{H}), 6.74$ (t, J = 7.4 Hz, 1H), $6.50(\mathrm{~d}, J=7.7 \mathrm{~Hz}, 2 \mathrm{H}), 5.50(\mathrm{~s}, 1 \mathrm{H}), 4.17(\mathrm{~s}$, 1H). ${ }^{13} \mathrm{C} \mathrm{NMR}\left(100 \mathrm{MHz}, \mathrm{CDCl}_{3}\right) \delta 147.69,146.50,140.22$, 133.96, 132.75, 129.33, 129.31, 128.94, 128.06, 118.64, 113.67, 111.55, 62.29. HRMS (MALDI-TOF) calculated for $\mathrm{C}_{20} \mathrm{H}_{15} \mathrm{ClN}_{2}$ $[\mathrm{M}+\mathrm{H}]^{+:}$319.1002, found: 319.0998 .

4-(Naphthalen-2-yl(phenylamino)methyl)benzonitrile (28): white solid, isolated yield: $91 \% .{ }^{1} \mathrm{H}$ NMR (400 $\left.\mathrm{MHz}, \mathrm{CDCl}_{3}\right) \delta$ 7.86-7.74 (m, 3H), 7.73 (s, 1H), 7.59 (d, $J=8.3 \mathrm{~Hz}, 2 \mathrm{H}), 7.53$ (d, $J$ $=8.3 \mathrm{~Hz}, 2 \mathrm{H}), 7.47(\mathrm{dd}, J=6.2,3.3 \mathrm{~Hz}, 2 \mathrm{H}), 7.38(\mathrm{dd}, J=8.5,1.5$ $\mathrm{Hz}, 1 \mathrm{H}), 7.13(\mathrm{t}, J=7.9 \mathrm{~Hz}, 2 \mathrm{H}), 6.74(\mathrm{t}, J=7.3 \mathrm{~Hz}, 1 \mathrm{H}), 6.54(\mathrm{~d}, J$ $=7.9 \mathrm{~Hz}, 2 \mathrm{H}), 5.67(\mathrm{~s}, 1 \mathrm{H}), 4.30(\mathrm{~s}, 1 \mathrm{H}) .{ }^{13} \mathrm{C}$ NMR $(100 \mathrm{MHz}$, $\left.\mathrm{CDCl}_{3}\right) \delta 148.14,146.88,139.16,133.48,133.06,132.72$, $129.39,129.19,128.26,128.10,127.82,126.67,126.60126 .52$, $125.42,118.84,118.51,113.74,111.37,63.14$. HRMS (MALDI-TOF) calculated for $\mathrm{C}_{24} \mathrm{H}_{18} \mathrm{~N}_{2}[\mathrm{M}-\mathrm{H}]^{+}: 333.1392$, found: 333.1388.

4-(((4-Fluorophenyl)amino)(phenyl)methyl)benzonitrile (29): pale yellow oil, isolated yield: 93\%. ${ }^{1} \mathrm{H}$ NMR (400 MHz, $\left.\mathrm{CDCl}_{3}\right) \delta 7.60(\mathrm{~d}, J=8.2 \mathrm{~Hz}, 2 \mathrm{H}), 7.50(\mathrm{~d}, J=8.2 \mathrm{~Hz}, 2 \mathrm{H})$, 7.36-7.27 (m, 5H), $6.81(\mathrm{t}, J=8.7 \mathrm{~Hz}, 2 \mathrm{H}), 6.49-6.38(\mathrm{~m}, 2 \mathrm{H})$, $5.45(\mathrm{~s}, 1 \mathrm{H}), 4.15(\mathrm{~s}, 1 \mathrm{H}) .{ }^{13} \mathrm{C}$ NMR $\left(100 \mathrm{MHz}, \mathrm{CDCl}_{3}\right) \delta 156.46$ (d, $J=237.35 \mathrm{~Hz}), 148.14,143.16(\mathrm{~d}, J=2.02 \mathrm{~Hz}), 141.76$, 132.72, 129.23, 128.23, 128.04, 127.61, 118.82, 115.80 (d, $J=$ $23 \mathrm{~Hz}$ ) 114.51 (d, $J=8.08 \mathrm{~Hz}$ ), 111.34, 63.52. ${ }^{19} \mathrm{~F}$ NMR (377 $\mathrm{MHz}, \mathrm{CDCl}_{3}$ ) $\delta-126.70$. HRMS (MALDI-TOF) calculated for $\mathrm{C}_{20} \mathrm{H}_{15} \mathrm{FN}_{2}[\mathrm{M}+\mathrm{H}]+:$ 303.1298, found: 303.1292 .

4-(((4-Chlorophenyl)amino) (phenyl)methyl)benzonitrile (30): pale yellow oil, isolated yield: 78\%. ${ }^{1} \mathrm{H}$ NMR $(400 \mathrm{MHz}$, $\left.\mathrm{CDCl}_{3}\right) \delta 7.61(\mathrm{~d}, J=8.3 \mathrm{~Hz}, 2 \mathrm{H}), 7.48(\mathrm{~d}, J=8.2 \mathrm{~Hz}, 2 \mathrm{H})$, 7.38-7.22 (m, 5H), $7.06(\mathrm{~d}, J=8.8 \mathrm{~Hz}, 2 \mathrm{H}), 6.42(\mathrm{~d}, J=8.8 \mathrm{~Hz}$, 2H), 5.47 (s, 1H), $4.26(\mathrm{~s}, 1 \mathrm{H}) .{ }^{13} \mathrm{C}$ NMR $\left(100 \mathrm{MHz}, \mathrm{CDCl}_{3}\right) \delta$ $147.80,145.33,141.47,132.76,129.28,129.19,128.33,128.05$, 127.64, 123.11, 118.77, 114.77, 111.45, 63.03. HRMS (MALDI-TOF) calculated for $\mathrm{C}_{20} \mathrm{H}_{15} \mathrm{ClN}_{2}[\mathrm{M}+\mathrm{Na}]^{+}:$341.0821, found: 341.0816.

4-(((4-Methoxyphenyl)amino)(phenyl)methyl)benzonitrile (31): pale yellow oil, isolated yield: $99 \% .{ }^{1} \mathrm{H}$ NMR $(400 \mathrm{MHz}$, $\left.\mathrm{CDCl}_{3}\right) \delta 7.62(\mathrm{~d}, J=8.3 \mathrm{~Hz}, 2 \mathrm{H}), 7.54(\mathrm{~d}, J=8.2 \mathrm{~Hz}, 2 \mathrm{H})$, 7.35-7.30 (m, 5H), $6.74(\mathrm{~d}, J=8.9 \mathrm{~Hz}, 2 \mathrm{H}), 6.48(\mathrm{~d}, J=8.9 \mathrm{~Hz}$, $2 \mathrm{H}), 5.46(\mathrm{~s}, 1 \mathrm{H}), 4.02(\mathrm{~s}, 1 \mathrm{H}), 3.72(\mathrm{~s}, 3 \mathrm{H}) .{ }^{13} \mathrm{C}$ NMR $(100 \mathrm{MHz}$, $\left.\mathrm{CDCl}_{3}\right) \delta 152.69,148.63,142.17,141.04,132.67,129.16$, 128.10. 128.07, 127.64, 118.90, 114.92, 114.88, 111.19, 63.79, 55.80. HRMS (MALDI-TOF) calculated for $\mathrm{C}_{21} \mathrm{H}_{18} \mathrm{~N}_{2} \mathrm{O}[\mathrm{M}]^{+}$: 314.1419, found: 314.1415.

4-(Phenyl((4-(trifluoromethyl)phenyl)amino)methyl)benzo nitrile (32): pale yellow oil, isolated yield: 47\%. ${ }^{1} \mathrm{H}$ NMR $(400$ $\left.\mathrm{MHz}, \mathrm{CDCl}_{3}\right) \delta 7.64(\mathrm{~d}, J=8.3 \mathrm{~Hz}, 2 \mathrm{H}), 7.49(\mathrm{~d}, J=8.2 \mathrm{~Hz}, 2 \mathrm{H})$, 7.41-7.29 (m, 5H), 7.27 (dd, $J=8.5,1.7 \mathrm{~Hz}, 2 \mathrm{H}$ ), 6.53 (d, $J=8.5$ $\mathrm{Hz}, 2 \mathrm{H}), 5.57$ (d, $J=4.1 \mathrm{~Hz}, 1 \mathrm{H}), 4.54(\mathrm{~d}, J=2.9 \mathrm{~Hz}, 1 \mathrm{H}) .{ }^{13} \mathrm{C} \mathrm{NMR}$ $\left(100 \mathrm{MHz} \mathrm{CDCl}_{3}\right) \delta 149.18,147.31,141.07,132.87,129.41$, $128.53,128.08,127.69,126.76(\mathrm{q}, J=4.04 \mathrm{~Hz}), 124.85(\mathrm{q}, J=$ $270.68 \mathrm{~Hz}), 120.18$ (q, $J=33.33 \mathrm{~Hz}) 118.70,112.98,111.71$, 62.63. $\left.{ }^{19} \mathrm{~F} \mathrm{NMR} \mathrm{(377} \mathrm{MHz,} \mathrm{CDCl}_{3}\right) \delta-61.22$. HRMS (MALDI-TOF) calculated for $\mathrm{C}_{21} \mathrm{H}_{15} \mathrm{~F}_{3} \mathrm{~N}_{2}[\mathrm{M}+\mathrm{H}]^{+}:$353.1266, found: 353.1260 .

\section{Results and discussion}

We initially tested our hypothesis by using benzaldehyde (1) and 1,4-dicyanobenzene (1,4-DCB, 2) as substrates, fac-Ir(ppy $)_{3}$ as photocatalyst and diisopropylethylamine (DIPEA) as the stoichiometric reductant in $\mathrm{CH}_{3} \mathrm{CN}$. To our delight, the desired benzhydrol was formed in 37\% yield under blue light irradiation for $12 \mathrm{~h}$ (Table 1 , entry 1). This encouraging result prompted us to optimize the efficiency of this reaction (Table 1). First, we carried out a solvent screen. When the reaction was performed in DMSO, the yield was dramatically enhanced to $82 \%$ (Table 1, entry 2). Other solvents such as acetone, DMF, 1,4-dioxane, DCM, DCE and $\mathrm{MeOH}$ showed lower efficiency than $\mathrm{CH}_{3} \mathrm{CN}$ (Table 1, entries 3-8). Replacing the iridium catalyst and electron donor with $\left[\mathrm{Ru}(\mathrm{bpy})_{3}\right] \mathrm{Cl}_{2}$ and Hantzsch ester, respectively, led to a sharp decrease in the yield (Table 1, entries 9 and 10). Triethylamine gave a similar yield to DIPEA (Table 1, entry 11), and increasing the loading of benzaldehyde and DIPEA did not further improve the yields of desired product (Table 1, entries 12 and 13). No product was obtained when DIPEA, visible light or photocatalyst were omitted, indicating that these three components were vital to the 
Table 1

Optimization of reaction conditions. a

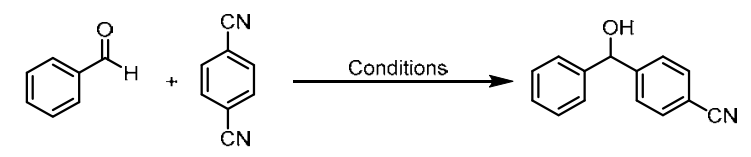

1

2

3

\begin{tabular}{llcc}
\hline Entry & Photocatalyst & Solvent & Yield ${ }^{\mathrm{b}}(\%)$ \\
\hline 1 & fac-Ir(ppy) $_{3}$ & $\mathrm{CH}_{3} \mathrm{CN}$ & 37 \\
2 & fac-Ir(ppy) $_{3}$ & DMSO & 82 \\
3 & fac-Ir(ppy) $_{3}$ & DMF & $<5$ \\
4 & fac-Ir(ppy) $_{3}$ & Acetone & 13 \\
5 & fac-Ir(ppy) $_{3}$ & 1,4-Dioxane & $<5$ \\
6 & fac-Ir(ppy) $_{3}$ & DCM & $<5$ \\
7 & fac-Ir(ppy) $_{3}$ & DCE & 6 \\
8 & fac-Ir(ppy) $_{3}$ & MeOH & $<5$ \\
9 & $\left.[\text { Ru(bpy) })_{3}\right]_{2}$ & DMSO & 0 \\
$10^{\mathrm{c}}$ & fac-Ir(ppy) $_{3}$ & DMSO & 29 \\
$11^{\mathrm{d}}$ & fac-Ir(ppy) $_{3}$ & DMSO & 80 \\
$12^{\mathrm{e}}$ & fac-Ir(ppy) $_{3}$ & DMSO & 82 \\
$13^{\mathrm{f}}$ & fac-Ir(ppy) $_{3}$ & DMSO & 82 \\
$14^{\mathrm{g}}$ & fac-Ir(ppy) $_{3}$ & DMSO & 0 \\
$15^{\mathrm{h}}$ & fac-Ir(ppy) $_{3}$ & DMSO & 0 \\
$16^{2}$ & None & DMSO & 0 \\
\hline
\end{tabular}

a Reaction conditions: Benzaldehyde $(0.3 \mathrm{mmol}), 1,4-\mathrm{DCB}(0.2 \mathrm{mmol})$, DIPEA (0.3 mmol), fac-Ir(ppy) 3 ( $2 \mathrm{~mol} \%$ ) blue LEDs irradiated for $12 \mathrm{~h}$ under an argon atmosphere. ${ }^{b}$ Detected by ${ }^{1} \mathrm{H}$ NMR analysis with diphenylacetonitrile as the internal standard. ${ }^{c}$ Hantzsch ester (HE, 0.3 mmol) as reductant. ${ }^{\mathrm{d}} \mathrm{Et}_{3} \mathrm{~N}(0.3 \mathrm{mmol})$ as reductant. ${ }^{\mathrm{e}}$ Benzaldehyde $(0.4$ mmol). ${ }^{\mathrm{D}}$ DIPEA (0.4 mmol). ${ }^{\mathrm{g}}$ No DIPEA. ${ }^{\mathrm{h}}$ No light.

performance of the reaction (Table 1, entries 14-16).

With the optimized conditions in hand, we applied this visible-light-catalyzed cross-coupling reaction to a range of molecules containing a $\mathrm{C}=\mathrm{X}$ bond $(\mathrm{X}=\mathrm{O}, \mathrm{N})$ and electron-deficient arenes to examine the scope. A variety of aryl nitriles afforded the corresponding cross-coupled products when benzaldehyde was used as the reaction partner, as shown in Scheme 2. A range of substituents, such as methyl, sulfuryl and ester groups, were tolerated well in this reaction (4-7). Aza-aromatic sub-

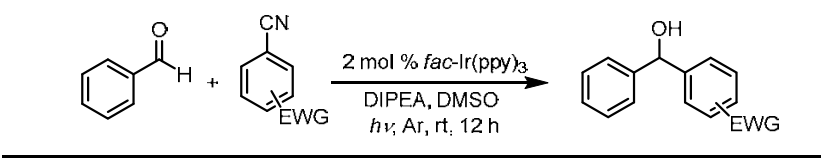

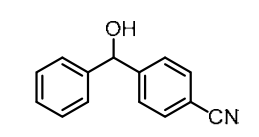

3. $83 \%$<smiles>COC(=O)c1ccc(C(O)c2ccccc2)cc1</smiles>

6. $48 \%$<smiles>OC(c1ccccc1)c1ccncc1</smiles>

$\mathbf{9}, 99 \%$<smiles>Cc1cc(C(O)c2ccccc2)ccc1C#N</smiles>

4, $82 \%$<smiles>O=C1OCc2cc(C(O)c3ccccc3)ccc21</smiles>

$7,42 \%$<smiles>Cc1cc(C(O)c2ccccc2)cc(C)n1</smiles>

$10,99 \%$
5. $52 \%$

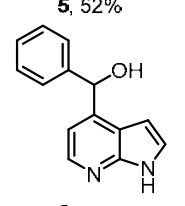

8, $82 \%$

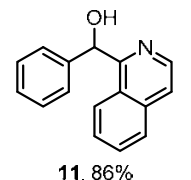

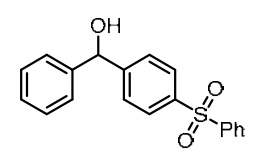

Scheme 2. Screening electron-deficient aryl nitrile substrates. strates, including 1H-pyrrolo[2,3-b]-pyridine-4-carbonitrile, isonicotinonitrile, 2,6-dimethyl- isonicotinonitrile and isoquinoline-1-carbonitrile, also worked well affording the corresponding products in yields 82\%-99\% (8-11).

We then explored the scope of the substrates containing the $\mathrm{C}=\mathrm{O}$ double bond (aldehydes and ketones) reacting with 1,4-DCB. The desired products were obtained in good to excellent yields. The results show that aldehydes with an electron-donating group had higher activity than those with electron-withdrawing groups $(\mathbf{1 2}, \mathbf{1 3}, \mathbf{1 6})$. Thiofuran and naphthalene were both compatible to give moderate yields $(\mathbf{1 4}, \mathbf{1 5})$. In sharp contrast to the aryl aldehyde, alkyl aldehydes were not reactive at all. Interestingly, ketones were found to be excellent reaction partners in this strategy, and showed higher reactivity than aldehydes (17). Under the optimized conditions, various substituted acetophenones and indanone afforded the desired products in 76\%-87\% yield (18-22). An acetophenone containing a cyclopropane group was also converted into the desired compound with preservation of three-membered ring (23).

Encouraged by the above results, we wanted to expand the scope of the new system by investigating the reactions of compounds containing a $\mathrm{C}=\mathrm{N}$ bond. To our delight, a range of aryl imines were active in this reaction, as shown in Scheme 4 . Numerous substitutions, including hydrogen, methyl, methoxy and chlorine all reacted well to give the desired products in 82\%-98\% yield (24-27). A naphthyl group was also tolerated to give $91 \%$ yield (28). When the para-proton of the aryl ring in $\mathrm{R}_{4}$ position was replaced with an electron-poor group, such as fluorine, chlorine or trifluoromethyl, the reactions gave moderate yields $(29, \mathbf{3 0}, \mathbf{3 2})$. The methoxy substituent at the same position gave an excellent yield of the desired product (31).

To further investigate the reaction process, radical trapping experiments were performed. When two equiv. of TEMPO were added into the template reaction, the yield of the desired prod-
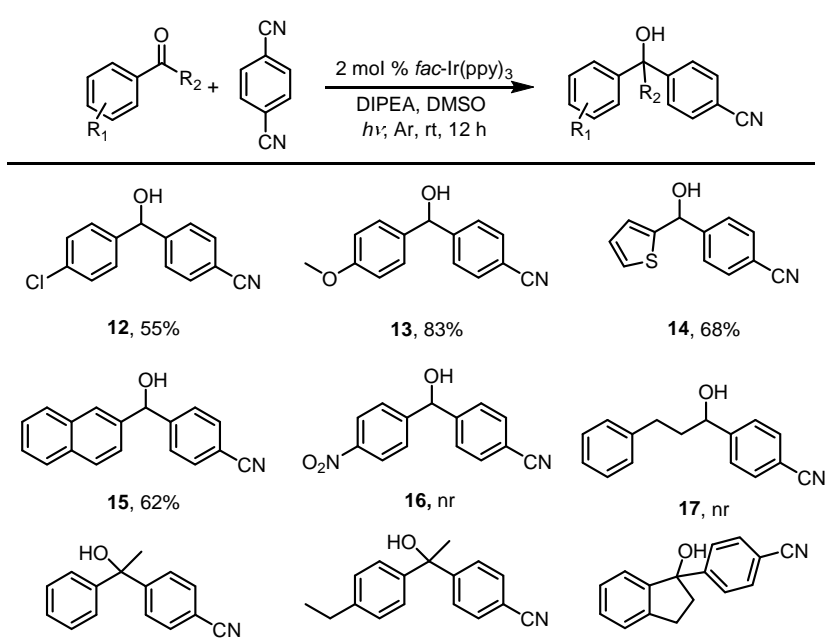

$14,68 \%$
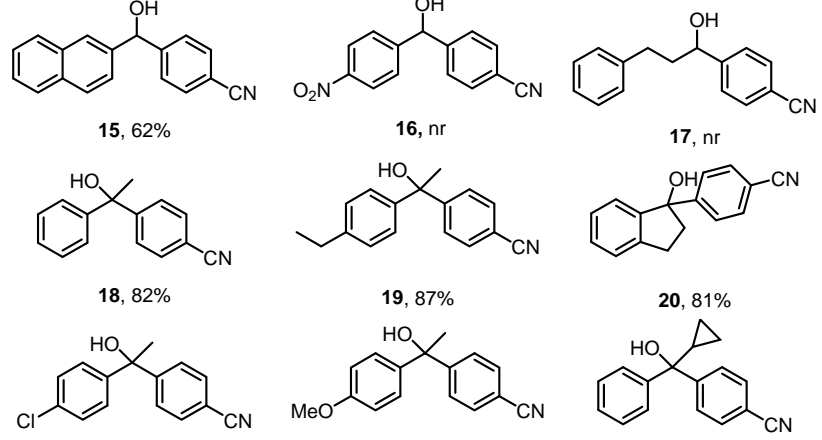

17, $\mathrm{nr}$
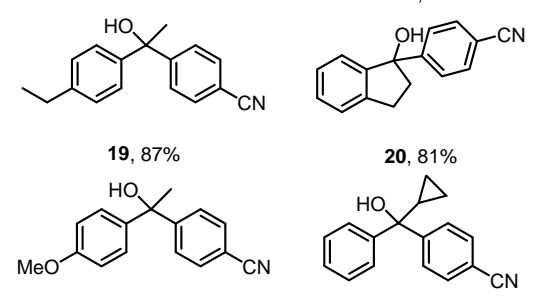

21, $76 \%$

22, $76 \%$

23, $67 \%$

Scheme 3. Screening aldehyde and ketone substrates. 


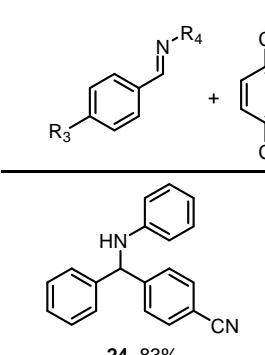

$\underset{\substack{\mathrm{DIPEA}, \mathrm{DMSO} \\ h v, \mathrm{Ar}, \mathrm{rt}, 12 \mathrm{~h}}}{2 \mathrm{~mol} \% \text { fac-Ir(ppy })_{3}} \longrightarrow$

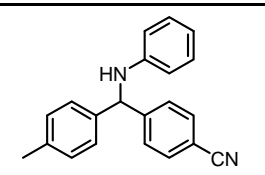

24, $83 \%$<smiles>N#Cc1ccc(C(Nc2ccccc2)c2ccc(Cl)cc2)cc1</smiles>

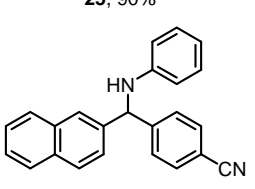

$27,82 \%$<smiles>N#Cc1ccc(C(Nc2ccc(Cl)cc2)c2ccccc2)cc1</smiles>

30, $78 \%$
25, $90 \%$
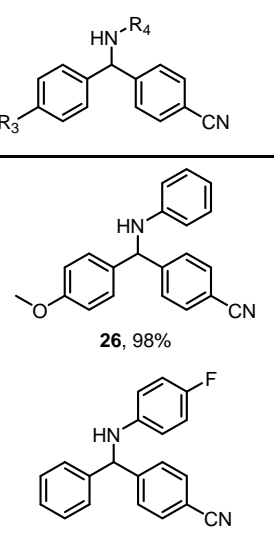

29, $93 \%$<smiles>COc1ccc(NC(c2ccccc2)c2ccc(C#N)cc2)cc1</smiles>

31, $99 \%$

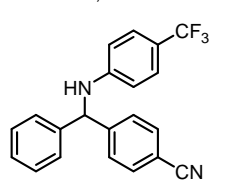

32, $47 \%$
Scheme 4. Screening imine substrates.

uct decreased dramatically from $82 \%$ to $19 \%$. This suggests the presence of a radical pathway in the reaction mechanism. Further details about the mechanism were determined by spec-
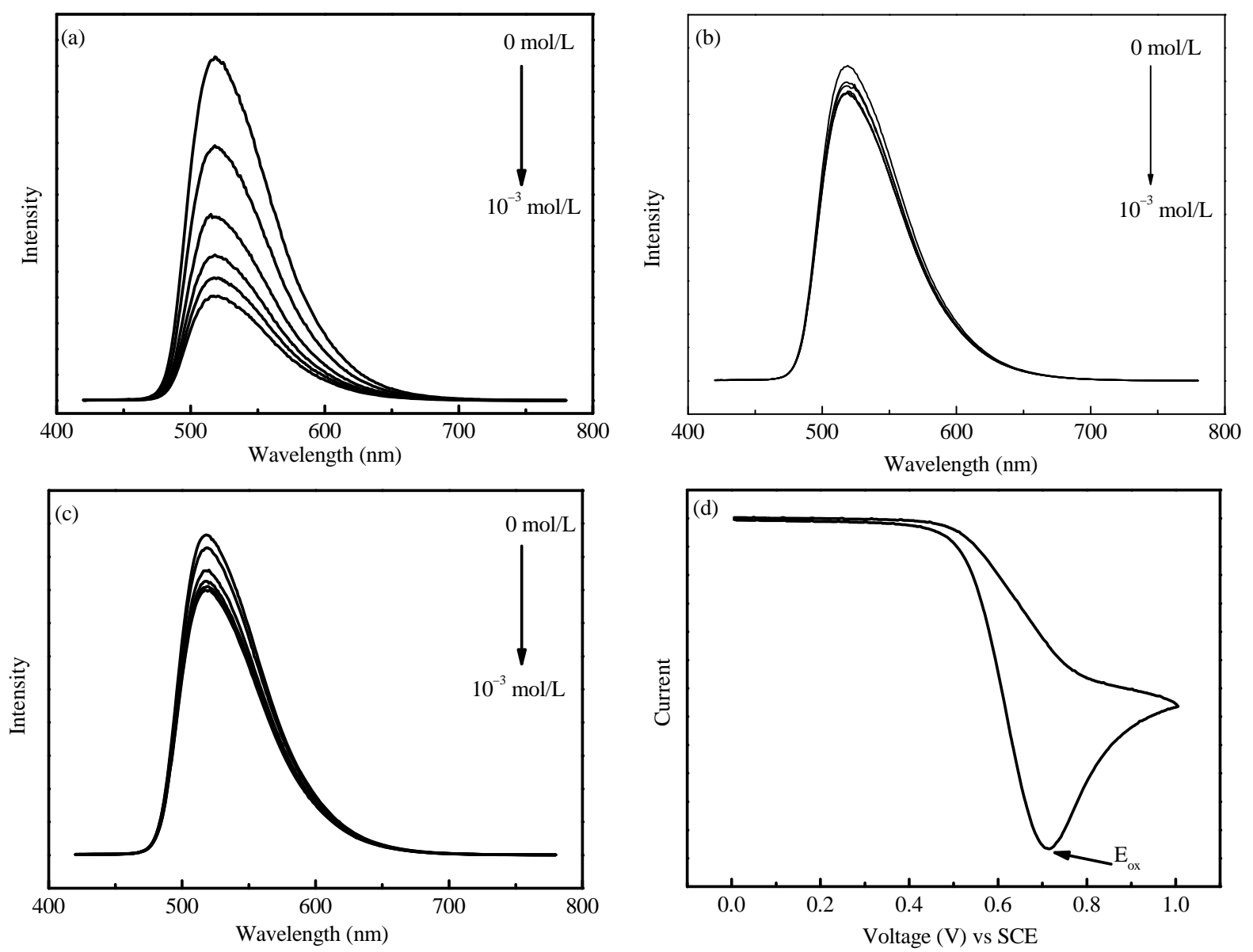

troscopic investigation. Quenching the excited iridium catalyst from the reactions involving 1,4-DCB, benzaldehyde and DIPEA (Fig. 1) demonstrated that 1,4-DCB was the only species to strongly interact with the excited $\operatorname{Ir}(\mathrm{ppy})_{3}$ [35-38]. However, the excited $\operatorname{Ir}(\mathrm{ppy})_{3}$ was not able to either reduce the aldehyde or oxidize the DIPEA because of the unfavorable redox potentials (reductive potentials for ${ }^{*} \operatorname{Ir}(\mathrm{ppy})_{3}$, aldehyde and 1,4-DCB are $-1.73,-1.93$ and $-1.61 \mathrm{~V}$, respectively vs SCE; oxidative potentials for $\operatorname{Ir}(\mathrm{ppy}) 3^{*}$ and DIPEA are 0.31 and $0.71 \mathrm{~V}$, respectively vs SCE) $[35,38,39]$. We therefore believe that this reaction begins with an interaction between $\operatorname{Ir}(\mathrm{ppy}) \mathrm{3}^{*}$ and 1,4-DCB.

Based on the above results and from previous reports [4,5], we proposed a plausible mechanism for this transformation (Scheme 5). Visible-light irradiation promotes the photosensitizer $f a c$-Ir(ppy) 3 to its excited state, a long-lived triplet state, which is oxidatively quenched by 1,4-DCB to afford the corresponding aryl radical anion $\mathbf{B}$ and oxidized iridium complex $\left[\operatorname{Ir}^{\mathrm{IV}}(\text { ppy })_{3}\right]^{+}\left(E_{1 / 2^{\mathrm{ox}}}=0.77 \mathrm{~V}\right)$. The generated $\mathrm{Ir}^{\mathrm{IV}}$ abstracts one electron from DIPEA to yield a DIPEA radical cation and regenerate the ground state $\operatorname{Ir}(\mathrm{ppy})_{3}$. Under the activation of this nitrogen radical cation, the $\mathrm{C}=0$ double bond is reduced by excited photocatalyst to afford the key radical intermediate $\mathbf{A}$, which subsequently couples with the aryl radical anion to furnish the desired product.

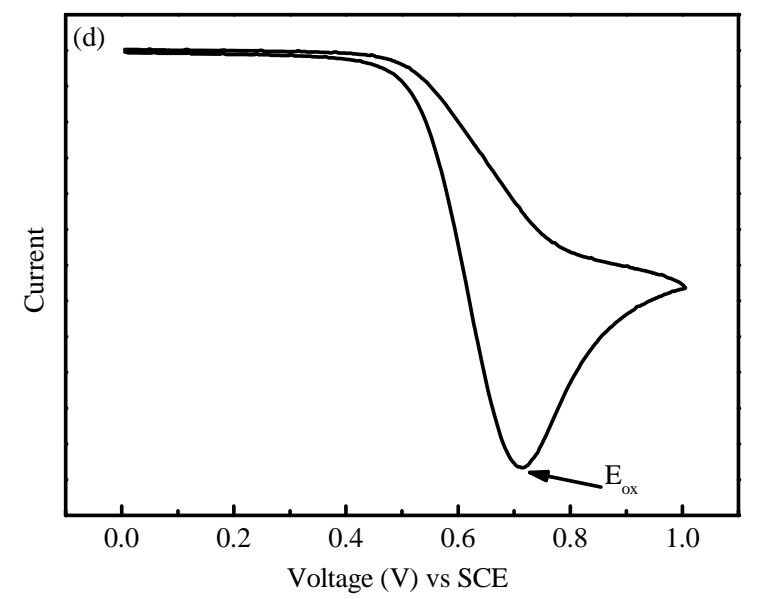

Fig. 1. Luminescence spectra of $\operatorname{Ir}(\text { ppy })_{3}$ as a function of concentration of 1,4-DCB (a), benzaldehyde (b) and DIPEA (c). [Ir(ppy) 3$]=1 \times 10^{-5} \mathrm{~mol} / \mathrm{L}$; solvent, DMSO; excitation wavelength, $400 \mathrm{~nm}$. (d) CV spectra of DIPEA. [DIPEA] $=10^{-2} \mathrm{~mol} / \mathrm{L}$. 


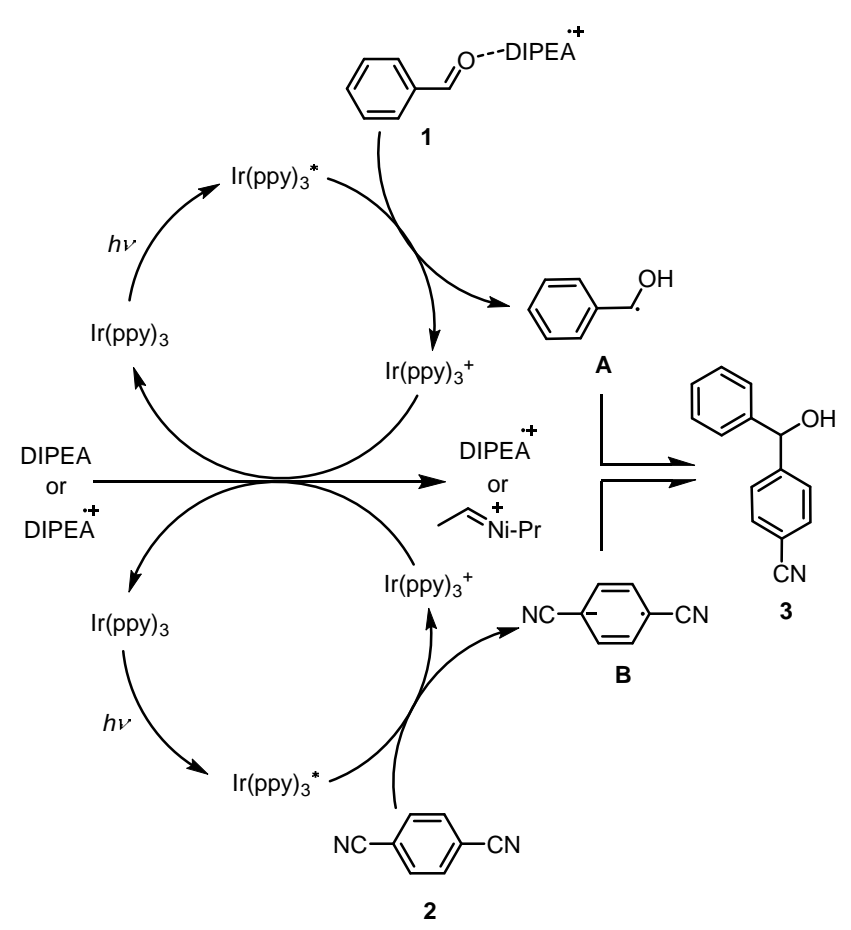

Scheme 5. Possible reaction mechanism.

\section{Conclusions}

In summary, we have successfully achieved the reductive radical-radical cross coupling of double bonds $(\mathrm{C}=\mathrm{O}$ and $\mathrm{C}=\mathrm{N})$ with electron-deficient aryl nitriles under mild reaction conditions. With a polarity inversion strategy induced by visible-light catalysis, various aryl substituted alcohols and amines have been constructed without involving air- or water-sensitive reagents. Broad substrate scope, high reaction yields and facile reaction conditions make this method a promising new approach for these important motifs. Further investigations into visible-light-induced polarity inversions are underway in our laboratory.

\section{Acknowledgments}

We thank Alison McGonagle, PhD, from Liwen Bianji, Edanz Editing China (www.liwenbianji.cn/ac), for editing the English text of a draft of this manuscript.

\section{References}

[1] D. A. Nicewicz, D. W. C. MacMillan, Science, 2008, 322, 77-80.

[2] M. A. Ischay, M. E. Anzovino, J. Du, T. P. Yoon, J. Am. Chem. Soc., 2008, 130, 12886-12887.

[3] J. M. R. Narayanam, J. W. Tucker, C. R. J. Stephenson, J. Am. Chem. Soc., 2009, 131, 8756-8757.

[4] D. C. Fabry, M. A. Ronge, J. Zoller, M. Rueping, Angew. Chem. Int. Ed., 2015, 54, 2801-2805.

[5] D. C. Fabry, J. Zoller, S. Raja, M. Rueping, Angew. Chem. Int. Ed., 2014, 53, 10228-10231.

[6] J. Du, T. P. Yoon, J. Am. Chem. Soc., 2009, 131, 14604-14605.

[7] M. A. Ischay, Z. Lu, T. P. Yoon, J. Am. Chem. Soc., 2010, 132, 8572-8574.

[8] C. J. Wallentin, J. D. Nguyen, P. Finkbeiner, C. R. J. Stephenson, J. Am. Chem. Soc., 2012, 134, 8875-8884.

[9] G. L. Lackner, K. W. Quasdorf, L. E. Overman, J. Am. Chem. Soc., 2013, 135, 15342-15345.

[10] D. Kalyani, K. B. McMurtrey, S. R. Neufeldt, M. S. Sanford, J. Am. Chem. Soc., 2011, 133, 18566-18569.

[11] X. Q. Hu, J. R. Chen, Q. Q. Zhao, Q. Wei, W. J. Xiao, X. T. Qi, Y. Lan, Nat. Commun., 2016, 7, 11188.

[12] J. D. Cuthbertson, D. W. C. MacMillan, Nature, 2015, 519, 74-77.

[13] C. P. Johnston, R. T. Smith, S. Allmendinger, D. W. C. MacMillan, Nature, 2016, 536, 322-325.

[14] X. Q. Huang, R. D. Webster, K. Harms, E. Meggers, J. Am. Chem. Soc., 2016, 138, 12636-12642.

[15] J. D. Nguyen, E. M. D'Amato, J. M. R. Narayanam, C. R. J. Stephenson, Nat. Chem., 2012, 4, 854-859.

[16] G. J. Choi, Q. L. Zhu, D. C. Miller, C. J. Gu, R. R. Knowles, Nature, 2016, 539, 268-271.

\section{Graphical Abstract}

Chin. J. Catal., 2018, 39: 487-494 doi: 10.1016/S1872-2067(17)62896-1

Photo-induced reductive cross-coupling of aldehydes, ketones and imines with electron-deficient arenes to construct aryl substituted alcohols and amines

Zan Liu, Xiaolei Nan, Tao Lei, Chao Zhou, Yang Wang,

Wenqiang Liu, Bin Chen, Chenho Tung, Lizhu Wu *

Technical Institute of Physics and Chemistry, Chinese Academy of Sciences; University of Chinese Academy of Sciences

Aryl-substituted alcohols and amines have been efficiently constructed by reductive radical-radical cross-coupling between aldehydes, ketones and imines with electron-deficient arenes under visible-light catalysis. The strategy of polarity inversion of the $\mathrm{C}=\mathrm{X}$ bond $(X=0, N)$ avoids the use of air- and water-sensitive reagents, tolerates various functional groups and produces the desired products in yields up to $99 \%$ at room temperature. 
[17] A. J. Musacchio, B. C. Lainhart, X. Zhang, S. G. Naguib, R. R. Knowles, T. C. Sherwood, Science, 2017, 355, 727-730.

[18] Q. Y. Meng, J. J. Zhong, Q. Liu, X. W. Gao, H. H. Zhang, T. Lei, Z. J. Li, K. Feng, B. Chen, C. H. Tung, L. Z. Wu, J. Am. Chem. Soc., 2013, 135, 19052-19055.

[19] G. T. Zhang, X. Hu, C. W. Chiang, H. Yi, P. K. Pei, A. K. Singh, A. W. Lei, J. Am. Chem. Soc., 2016, 138, 12037-12040.

[20] J. Schwarz, B. Konig, Green Chem., 2016, 18, 4743-4749.

[21] X. Y Yu, F Zhou, J. R. Chen, W. J. Xiao, Acta Chim. Sin., 2017, 75, 86-91.

[22] A. R. Wade, H. R. Pawar, M. V. Biware, R. C. Chikate, Green Chem., 2015, 17, 3879-3888.

[23] X. J. Lang, J. C. Zhao, X. D. Chen, Angew. Chem. Int. Ed., 2016, 55, 4697-4700.

[24] T. Ikeda, S. Yue, C. R. Hutchinson, J. Org. Chem., 1985, 50, 5193-5199.

[25] M. Szostak, D. J. Procter, Angew. Chem. Int. Ed., 2012, 51, 9238-9256.

[26] L. J. Rono, H. G. Yayla, D. Y. Wang, M. F. Armstrong, R. R. Knowles, J. Am. Chem. Soc., 2013, 135, 17735-17738.

[27] K. T. Tarantino, P. Liu, R. R. Knowles, J. Am. Chem. Soc., 2013, 135, 10022-10025.

[28] J. L. Jeffrey, F. R. Petronijević, D. W. C. MacMillan, J. Am. Chem. Soc.,
2015, 137, 8404-8407.

[29] F. R. Petronijević, M. Nappi, D. W. C. MacMillan, J. Am. Chem. Soc., 2013, 135, 18323-18326.

[30] M. Nakajima, E. Fava, S. Loescher, Z. Jiang, M. Rueping, Angew. Chem. Int. Ed., 2015, 54, 8828-8832.

[31] E. Fava, A. Millet, M. Nakajima, S. Loescher, M. Rueping, Angew. Chem. Int. Ed., 2016, 55, 6776-6779.

[32] W. Ding, L. Q. Lu, J. Liu, D. Liu, H. T. Song, W. J. Xiao, J. Org. Chem., 2016, 81, 7237-7243.

[33] L. Qi, Y. Y. Chen, Angew. Chem. Int. Ed., 2016, 55, 13312-13315.

[34] K. N. Lee, Z. Lei, M. Y. Ngai, J. Am. Chem. Soc., 2017, 139, 5003-5006.

[35] A. McNally, C. K. Prier, D. W. C. MacMillan, Science, 2011, 334, 1114-1117.

[36] M. T. Pirnot, D. A. Rankic, D. B. C. Martin, D. W. C. MacMillan, Science, 2013, 339, 1593-1596.

[37] K. Qvortrup, D. A. Rankic, D. W. C. MacMillan, J. Am. Chem. Soc., 2014, 136, 626-629.

[38] H. G. Roth, N. A. Romero, D. A. Nicewicz, Synlett, 2016, 27, 714-723.

[39] C. K. Prier, D. A. Rankic, D. W. C. MacMillan, Chem. Rev., 2013, 113, 5322-5363.

\title{
可见光催化醛、酮或亚胺与缺电子芳烃还原偶联构筑芳基取代醇和胺
}

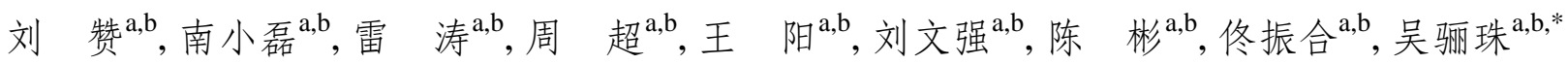 \\ a中国科学院理化技术研究所, 光化学转换与功能材料重点实验室, 北京 100190 \\ b中国科学院大学, 北京100049
}

摘要: 通过 $C=X(X=O, N)$ 双键极性翻转构筑碳-碳键是有机化学反应的重要合成策略. 传统 $C=X(X=O, N)$ 双键的极性翻转 往往需要苛刻的反应条件和对水或空气敏感的强还原剂辅助, 导致其适用范围受限. 近年来, 可见光催化反应以其独特高 效的单电子转移特性, 在室温条件下实现了这一类贫电子官能团向亲核性中间体的高效转化. 该策略已经拓展 $C=X(X=O$, $\mathrm{N})$ 双键自身或与烷基链的偶联, 从而得到烷基取代的醇和胺类化合物. 本文利用可见光催化反应使 $C=X(X=O, N)$ 双键极 性翻转与芳香化合物的直接偶联, 高效温和地合成芳基取代的醇和胺. 反应无需强还原剂, 底物适用范围广. 该方法是对 可见光催化 $C=X(X=O, N)$ 双键极性翻转的重要补充, 具有潜在的应用价值.

本文以苯甲醛和1,4-二㲵基苯为底物, $f a c-I r(p p y)_{3}$ 为光敏剂, 二异丙基乙胺为终端还原剂, DMSO为溶剂, 蓝光照射 $12 \mathrm{~h}$ 能够以 $82 \%$ 的收率实现模板反应. 其它光敏剂如 $\left[\mathrm{Ru}(\mathrm{bpy})_{3}\right] \mathrm{Cl}_{2}$ 则不能催化该反应. 溶剂效应指出, 丙酮、乙腈可以得到低于 40\%的收率, 甲醇、二氯甲烷、DMF等溶剂不适用该反应体系. 控制实验证实, 光敏剂、二异丙基乙胺和光照三个反应组 分缺一不可. 底物拓展发现, 不同取代基的芳基腈类化合物包括烷基取代、砜基和酯基取代甚至杂芳环取代都能很好地适 用于该体系, 芳基醛、酮以及亚胺作为反应的另一组分亦能高效参与该还原偶联反应.

自由基捕获实验证实反应过程中涉及自由基历程. 光谱淬灭实验表明, 芳香腈是唯一有效淬灭激发态 $f a c-I r(p p y)_{3}$ 发光 的物种. 进一步结合底物的氧化还原电位, 证实芳香腈能被激发态的光敏剂 fac-Ir(ppy) 还原, 但二异丙基乙胺和芳香醛、酮 不能与激发态光敏剂发生作用, 催化反应经历光敏剂的氧化淬灭路径. 首先, 光敏剂受光激发到达激发态, 与芳基腈发生 单电子转移. 随后, 二异丙基乙胺促使失去电子的铱配合物还原再生, 得到相应氮自由基阳离子. 该氮自由基阳离子活化 反应体系中的 $\mathrm{C}=\mathrm{X}(\mathrm{X}=\mathrm{O}, \mathrm{N})$ 双键, 使其从激发态铱物种得到电子形成芐位自由基, 进而与得到电子的芳基氰偶联得到最终 产物.

关键词: 芳基取代的醇和胺; 还原偶联; 极性翻转; 光催化; 芳基化

收稿日期: 2017-09-17. 接受日期: 2017-10-26. 出版日期: 2018-03-05.

*通讯联系人. 电话/传真: (010)82543580; 电子邮箱: lzwu@mail.ipc.ac.cn

基金来源：国家科技部基金(2013CB834804，2014CB239402); 国家自然科学基金(21390404, 91427303); 中国科学院战略先导研 究项目基金(XDB17030400).

本文的英文电子版由Elsevier出版社在ScienceDirect上出版(http://www.sciencedirect.com/science/journal/18722067). 